\title{
EFFICACY OF BARIATRIC SURGERY IN OBESE INDIAN WITH TYPE 2 DIABETES MELLITUS
}

Subodh Banzal, Abhishek Singhai, Mohit Bhandari, Padmnabh Sharma, Rajesh Kumar Jha, Piyush Manoria

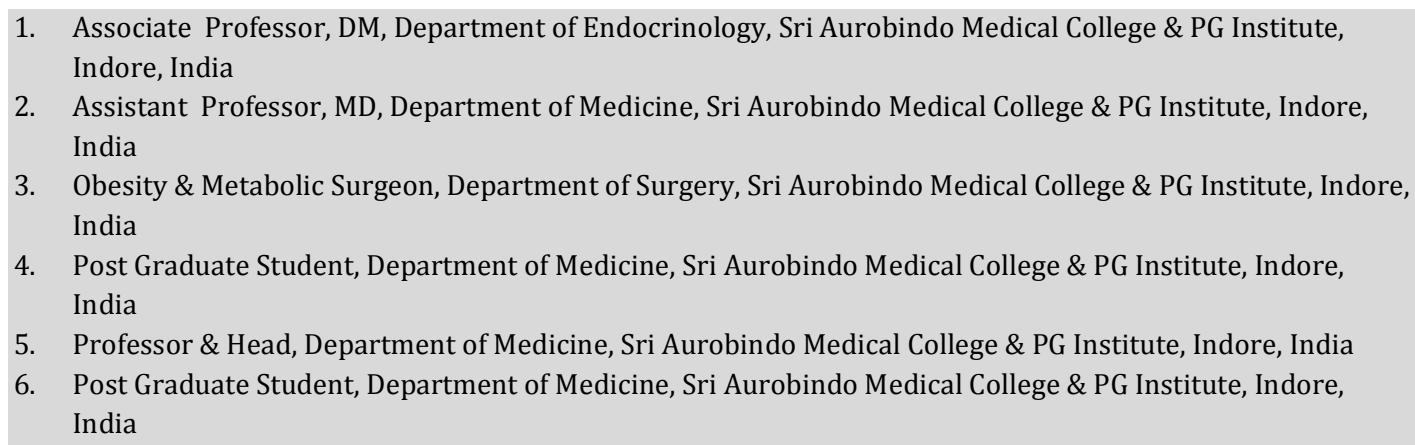

\section{CORRESPONDING AUTHOR}

Dr. Abhishek Singhai,

MD Medicine, Assistant Professor,

Department of Medicine,

Sri Aurobindo Medical College \& PG Institute,

Indore, India

E-mail: drabhisheksinghai@gmail.com

Ph: 00919926597070.

ABSTRACT: CONTEXT: Individuals with morbid obesity and type 2 diabetes benefit from weight loss, as this allows better glycemic control and modifies the coexisting risk factors for coronary heart disease, namely hypertension, dyslipidemia, insulin resistance, sleep apnea, and other comorbidities that constitute the metabolic syndrome. AIMS: The purpose of the present study was to investigate whether weight loss after bariatric surgery can correct glycemic control and reduces the need of anti-diabetic treatment in morbidly obese patients with type 2 diabetes. SETTINGS AND DESIGN: This is a prospective cohort study performed in Sri Aurobindo Medical College \& PG Institute, Indore. Sampling done was nonrandom and purposive. METHODS AND MATERIAL: Forty patients with body mass index (BMI) > 35 and had known type 2 diabetes were enrolled in study, all these patient undergone bariatric surgery. Their obesity status in terms of height, weight and BMI, Glycemic status in terms of fasting blood sugar (FBS), postprandial blood sugar (PPBS) and glycosylated haemoglobin (HbA1c), and treatment status in terms of oral hypoglycemic agents and insulin were noted in details preoperatively Statistical analysis used: Quantitative variables were tested using Chi square test and $p$ values were calculated between two groups. $p$ value of $\leq 0.05$ was considered statistically significant. Averages were expressed between groups as mean \pm standard deviation or percentage. RESULTS: Our study shows good control of glycemic status after bariatric surgery with mean HbA1c within desired level after 6 months of bariatric surgery. CONCLUSIONS: In conclusion, our study shows that bariatric surgery is an effective option for morbidly obese patients with type 2 diabetes mellitus. Weight loss due to surgery is strongly associated with good glycemic control and improved treatment efficacy.

KEY-WORDS: Type 2 diabetes, obesity, bariatric surgery 
INTRODUCTION: Epidemiological studies have shown that obesity is a significant risk factor for type 2 diabetes and that $41 \%$ of morbidly obese patients have abnormal glycemic control.(1) Insulin resistance is more pronounced in obese subjects with type 2 diabetes. ${ }^{(2,3)}$ It has been shown that progression from normal to impaired glucose tolerance and diabetes is associated with a reduction in insulin sensitivity and a progressive decrease of the acute insulin response to glucose, which is lost at the onset of diabetes. $(4,5)$ Moderate weight loss following a low caloric diet can improve insulin action and secretion, but the effect is rather weak.(6) Metabolic control has been shown to improve the defect of early insulin response only minimally or partially. ${ }^{(7,8)}$ Individuals with morbid obesity and type 2 diabetes benefit from weight loss, as this allows better glycemic control and modifies the coexisting risk factors for coronary heart disease, namely hypertension, dyslipidemia, insulin resistance, sleep apnea, and other comorbidities that constitute the metabolic syndrome (9) Although weight loss can be achieved by low-calorie diet, exercise, behaviour modification, and medical treatment, late weight gain has been an almost universal problem. Conversely bariatric surgeries maintains a weight loss of $33 \%$ of body weight for $>10$ years, a result associated with normalization of glucose levels in most patients with impaired glucose tolerance and type 2 diabetes.(10) It also normalizes insulin action and secretion in normal glucose tolerant subjects with morbid obesity.(11,12) To our knowledge, there are no other anti-diabetic therapies that can produce such effective and longterm glycemic control.

The purpose of the present study was to investigate whether weight loss after bariatric surgery can correct glycemic control and reduces the need of anti-diabetic treatment in morbidly obese patients with type 2 diabetes.

SUBJECTS AND METHODS: This is a prospective cohort study performed in Sri Aurobindo Medical College \& PG Institute, Indore. Sampling done was nonrandom and purposive. Study was performed after consent from subjects and approval of ethical committee, the data's was kept anonymous. Forty patients with BMI $>35$ and had known type 2 diabetes were enrolled in study. All these patient undergone bariatric surgery (sleeve gastrectomy) after proper preanesthetic evaluation. Their obesity status in terms of height, weight and BMI, Glycemic status in terms of FBS, PPBS and HbA1c, and treatment status in terms of oral hypoglycemic agents and insulin were noted in details preoperatively.

Patients were then discharged and advised to strictly follow dietary advices and to frequently follow endocrinologists. The patients were then followed after three months and six months respectively. The obesity, glycemic and treatment status were again noted in detail on both occasions. Any chronic complications of diabetes mellitus if developed were also noted. Inclusion criteria:

- Adult patient with known type 2 diabetes mellitus

- Obese with BMI $>35 \mathrm{~kg} / \mathrm{m}^{2}$

- Subject undergoing bariatric surgery

Exclusion criteria:

- $\mathrm{BMI}<35 \mathrm{~kg} / \mathrm{m}^{2}$

- Patients with known chronic complications of diabetes mellitus 2

- Patients who developed chronic complication of diabetes mellitus 2 during follow up 
RESULTS: The mean body weight which was recorded pre operatively was $111.95 \pm 46.2$ (mean $\pm 2 \mathrm{SD}$ ). All the 40 patients shows significant weight reduction after 3 months surgery during follow up the mean body weight of subjects were $99.2 \pm 33.8$ with reduction of $12.75 \mathrm{~kg}(\mathrm{p}<$ 0.006 ) and after 6 months there is further mean weight reduction of $12.55 \mathrm{~kg}$ from that of at 3 months $(p<0.05)$.In terms of BMI, the mean BMI of subjects at initial study was $44.42 \pm 14.54$ which shows a substantial reduction of 6.52 mean value when patients were followed up after 3 months $(\mathrm{p}<0.003)$ there is mean reduction of 5.36 after 3 months of first follow up ( $<<$ 0.003).(Figure.1) Most of the patients show uncontrolled glycemic status preoperatively even they are on anti-diabetic treatment, patients are advised to follow up regularly for better control and treatment of their diabetes and glucose status respectively. The mean value of $\mathrm{HbA1c}$ at the initiation of study was $6.825 \%$ which shows a good response with reduction of 0.55 of mean value $(\mathrm{p}=0.003)$ after 3 months and the value further reduces 0.415 after next 3 months $(\mathrm{p}<$ 0.009). (Figure.2) The FBS and PPBS of patients were noted, the mean FBS and PPBS preoperatively was $128 \pm 34$ and $196.35 \pm 55$ respectively which shows poor control of diabetes. FBS mean value reduced to $102.15 \pm 21$ and $95.4 \pm 14$ at 3 and 6 months follow up ( $p<$ $0.009)$ respectively. PPBS mean values also reduced to $138.08 \pm 27.4(\mathrm{p}<0.001)$ after 3 months and $128.85 \pm 16.66(\mathrm{p}=0.20)$ at 6 months. The PPBS values at 6 months did not reach any statistically significant value; else FBS and PPBS shows reduction in mean values during follow up.

Out of 40 patients, 14 patients were on oral hypoglycemic agents (OHA) and 26 were on insulin in different doses. Out of 14 patients who were on OHA, 4 were on triple drug therapy (metformin + sulfonylurea + pioglitazone) and rest 10 patients were on double drugs (metformin + sulfonylurea). After 3 months patients on triple drug therapy were put on two drugs on the basis of their needs. Two patients required metformin only after 6 months while other continued to be on two drugs (metformin + sulfonylurea). Out of 10 patients who were on two drugs (metformin + sulfonylurea), 6 patients weaned off from drugs after 6 months and are advised on life style modification only. Out of remaining 4 patients, 2 patients were put on metformin only after 6 months and the two were put on same two drug regime but with reduced dose.

Out of 26 patients who were taking insulin, 6 were on human premix insulin. Mean insulin demand decreased by 18 units in these patients 3 months after bariatric surgery and by 10 units in next three months. In remaining 20 patients, 12 were on detemir and aspart combination (long acting + ultra-short acting) and 8 were on detemir and regular insulin combination (long acting + short acting). Out of 12 patients who were on detemir and aspart insulin combination, 2 patients were switched over to detemir plus OHA combination. Insulin demand decreased in remaining 10 patients, mean decrease of 13.2 units aspart insulin noted in six months in which mean reduction of 6 units occurs in first 3 months and of 7.2 units in next 3 months. Out of 8 patients who were on detemir and regular insulin combination, 2 patients were switched to OHA and remaining 6 patients showed improvement in their insulin need as there is reduction of mean 16 units of regular insulin after 6 months of operation. Out of 20 patients who were on detemir along with short acting insulin 6 patients were kept on same dose, while other shows reduction in dose of detemir also, there is mean reduction of 4 units of detemir in 6 months. (Figure.3)

DISCUSSION: The procedure bariatric surgery was successful in all patients, and weight reduction was impressive, although the patients were still obese 6 months after the operation, 
with an average BMI $\sim 30 \mathrm{~kg} / \mathrm{m}^{2}$. In our study we found that patients showed marked improvement in their diabetic status. While 3 patients showed complete remission of diabetes mellitus, others also show significant decrease in their insulin or OHA demand.

A systematic review and meta-analysis of the English literature reported complete resolution of type 2 diabetes (defined as discontinuation of all diabetes-related medications and blood glucose levels within the normal range) in $78.1 \%$ of cases. This percentage increased to 86.6\% when counting patients reporting improvement of glycemic control, and diabetes resolution occurred in concomitance with an average weight loss of $38.5 \mathrm{~kg}$ (55.9\% of the excess weight).(13)

Two large case-series studies, by Pories et al.(14) (330 patients) and Schauer et al.(15), focused principally on diabetes outcomes after bariatric surgery. In the former study, mean FBG decreased from clearly diabetic values to near normal levels (117 $\mathrm{mg} \%)$, and $\mathrm{HbA}_{1 \mathrm{c}}$ fell to normal levels $(6.6 \%)$ without diabetes medicines in $89 \%$ of patients. In the latest study by Schauer et al., researchers provided the in-depth evaluation of the clinical outcome in 240 diabetic morbidly obese bariatric patients with a follow-up rate of $80 \%$. The authors noted that after surgery, weight and BMI decreased from $308 \mathrm{lbs}$ and $50.1 \mathrm{~kg} / \mathrm{m}^{2}$ to $211 \mathrm{lbs}$ and $34 \mathrm{~kg} / \mathrm{m}^{2}$ for a mean weight loss of $97 \mathrm{lbs}$ and mean excess weight loss of $60 \%$. Fasting plasma glucose and $\mathrm{HbA}_{1 \mathrm{c}}$ concentrations returned to normal levels (in 83\%) or markedly improved (in 17\%) in all patients. A significant reduction in use of oral antidiabetic agents (80\%) and insulin (79\%) followed surgical treatment. Patients with the shortest duration ( $<5$ years), the mildest form of type 2 diabetes (diet controlled), and the greatest weight loss after surgery were most likely to achieve complete resolution of type 2 diabetes.

Two prospective, controlled studies have addressed changes in glycemic control after bariatric surgery. The multicenter Swedish Obese Subjects (SOS) study compared bariatric surgery with medical weight-loss treatment in well-matched obese patients.(16) Bariatric surgery caused an average $16.1 \%$ weight loss at 10 years, compared with a small weight gain in control subjects. Mean FBG tended to increase during the study in nonsurgical controls $(+18.7 \%$ at 10 years), whereas a substantial decrease was seen in surgical patients at 2 years $(-13.6 \%)$ and 10 years $(-2.5 \%)$. The risk of developing diabetes was more than three times lower for surgically treated patients at 10 years, and recovery rates from diabetes were three times greater. Dixon et al.(17) reported a randomized controlled trial comparing bariatric surgery to conventional type 2 diabetes management in subjects with BMI $30-40 \mathrm{~kg} / \mathrm{m}^{2}$ who had early $(<2$ years duration) and relatively mild diabetes. Bariatric surgery promoted significantly larger reductions in FBG, $\mathrm{HbA}_{1 \mathrm{c}}$, and diabetes medication usage. Scopinaro et al. (18) showed a stunning $97 \%$ euglycemia in 268 diabetic patients at 10 years after surgery.

There is now enough evidence to state that bariatric surgery may reduce mortality in patients with diabetes. In the analysis by Adams et al.(19), deaths attributed to diabetes were reduced by $92 \%$. Thus, there can be little doubt that in very obese patients with type 2 diabetes, bariatric surgery in general is a highly effective means of treating type 2 diabetes.

We closely observe that patient who shows remission are those who had shorter duration of diabetes mellitus and had significant amount of weight loss postoperatively, also all the patients with comparatively more weight loss and shorter duration of diabetes mellitus shows more reduction in their insulin demands.

CONCLUSION: Our study shows that bariatric surgery is an effective option for severely obese patients with type 2 diabetes mellitus and weight loss due to surgery is strongly associated with 
good glycemic control and improved treatment efficacy. Our study also supports the concept that insulin resistance is major determinant during initial and early years of type 2 diabetes mellitus and due to weight loss their occurs the improvement in insulin sensitivity which results in remission to patients in initial years of type 2 diabetes mellitus.

\section{REFERENCES:}

1. Buffington CK, Cowan GS Jr. Gastric bypass in the treatment of diabetes, hypertension and lipid/lipoprotein abnormalities of the morbidly obese. In Update: Surgery for the Morbidly Obese Patient. Deitel M, Cowan GS Jr, Eds. Toronto, FD-Communications, 2000, p. 435-449

2. Kahn BB, Flier JS. Obesity and insulin resistance. J Clin Invest 2000;106:473- 481

3. De Fronzo RA. Pathogenesis of type 2 diabetes: metabolic and molecular implications for identifying diabetes genes. Diabetes Rev 1997;5:177-269

4. Brunzell JD, Robertson RP, Lerner RL, Hazzard WR, Ensinck JW, Bierman EL, et al. Relationship between fasting plasma glucose levels and insulin secretion during intravenous glucose tolerance tests. J Clin Endocrinol Metab 1976;42:222-229

5. Weir GC, Bonner-Weir S. Insulin secretion in non-insulin-dependent diabetes mellitus. In Diabetes Mellitus. 2nd ed. Le Roith D, Taylor SI, Olefsky JM, Eds. Philadelphia, Lippincott, Williams and Wilkins 2000, p. 595-603

6. Weyer C, Hanson K, Bogardus C, Pratley RE. Long term changes in insulin action and insulin secretion associated with gain, loss, regain and maintenance of body weight. Diabetologia 2000;43:36-46

7. Garvey WT, Olefsky JM, Hamman RF, Kolterman OG. The effect of insulin treatment on insulin secretion and insulin action in type II diabetes mellitus. Diabetes 1985;34:222234

8. Vague P, Moulin JP. The defective glucose sensitivity of the beta-cell in insulin dependent diabetes: improvement after twenty hours of normoglycaemia. Metabolism 1982;31:139-142

9. Cowan GS Jr, Buffington CK. Significant changes in blood pressure, glucose and lipids with gastric bypass surgery. World J Surg 1998;22:987-992

10. Kalfarentzos F, Dimakopoulos A, Kehagias I, Loukidi A, Mead N. Vertical banded gastroplasty versus standard or distal Roux-en-Y gastric bypass based on specific selection criteria in the morbidly obese: preliminary results. Obes Surg 1999;9:433-42

11. Lextiexhe MR, Scheen AJ, Gerard PL, Desaive C, Lefebvre PJ. Postgastroplasty recovery of ideal body weight normalizes glucose and insulin metabolism in obese women. J Clin Endocrinol Metab1995;80:364-369

12. Scheen AJ, Paquot N, Letiexhe MR, Paolisso G, Castillo MJ, Lefebvre PJ. Glucose metabolism in obese subjects: lessons from OGTT, IVGTT and clamp studies. Int J Obes 1995;S14-S20

13. Buchwald H, Estok R, Fahrbach K. Weight and type 2 diabetes after bariatric surgery: systematic review and meta-analysis. Am J Med 2009;122:248-256

14. Pories WJ, Swanson MS, MacDonald KG. Who would have thought it? An operation proves to be the most effective therapy for adult-onset diabetes mellitus. Ann Surg 1995;222:339-350

15. Schauer PR, Burguera B, kramuddin S. Effect of laparoscopic Roux-en Y gastric bypass on type 2 diabetes mellitus. Ann Surg 2003;238:467-484

16. Sjöström L, Lindroos AK, Peltonen M. Swedish Obese Subjects Study Scientific Group. Lifestyle, diabetes, and cardiovascular risk factors 10 years after bariatric surgery. $\mathrm{N}$ Engl J Med 2004;351:2683-2693

17. Dixon JB, O'Brien PE, Playfair J, et al. Laparoscopic adjustable gastric banding in severely obese adolescents: a randomized trial. JAMA 2008;299:316-323 
18. Marinari GM, Papadia FS, Briatore L, Adami G, Scopinaro N. Type 2 diabetes and weight loss following biliopancreatic diversion for obesity. Obes Surg 2006;16:1440-1444

19. Adams TD, Gress RE, Smith SC, Halverson RC, Simper SC, Rosamond WD, et al. Long-term mortality after gastric bypass surgery. N Engl J Med 2007;357:753-761

Figure.1: Weight and BMI changes preoperatively, 3 months and 6 months after bariatric surgery

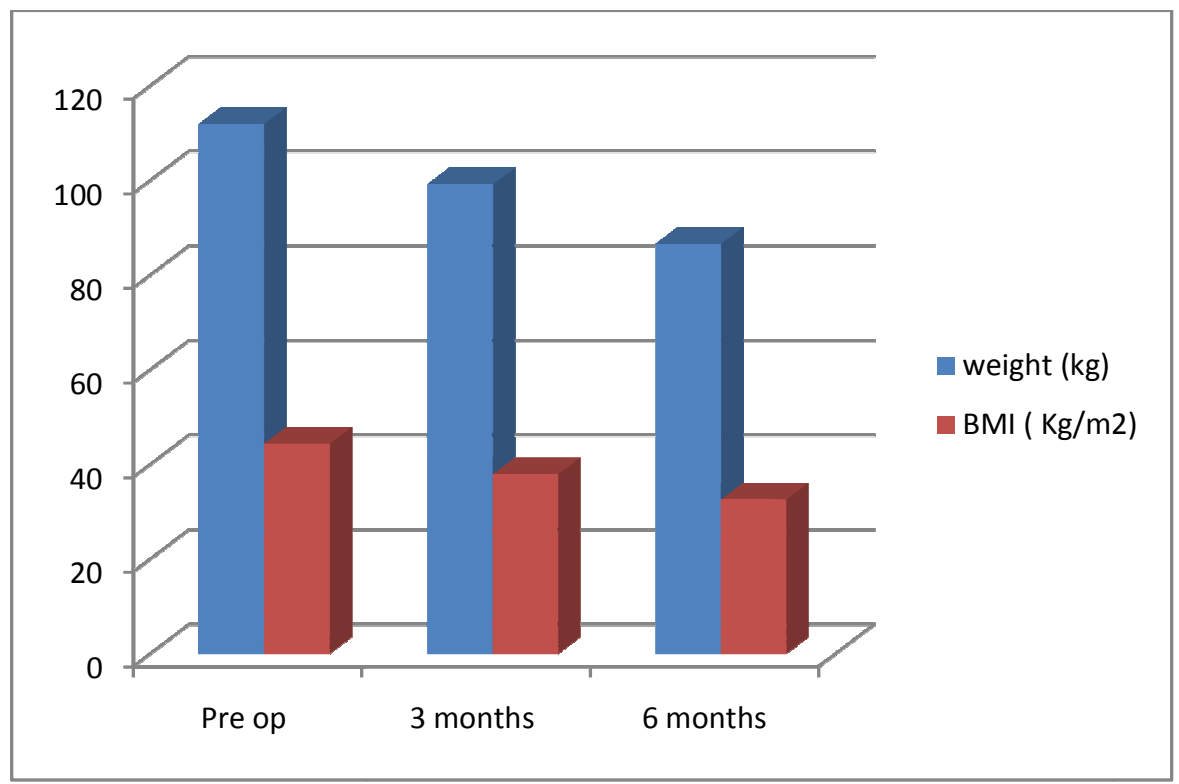

Figure.2: HbA1c level preoperatively, 3 months and 6 months after bariatric surgery

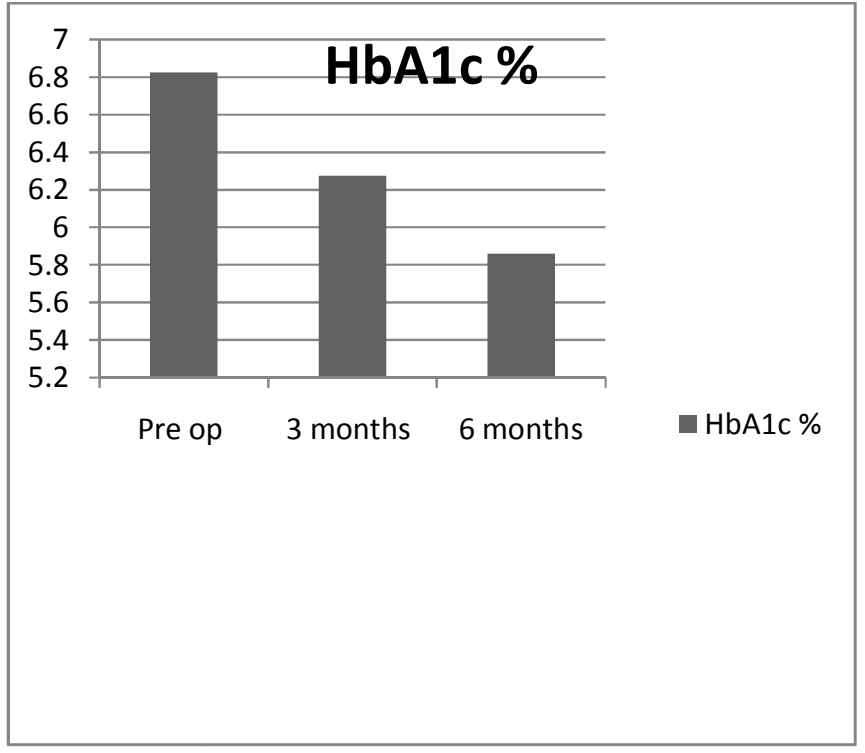


Figure.3: Mean Insulin dose preoperatively, 3 months and 6 months after bariatric surgery

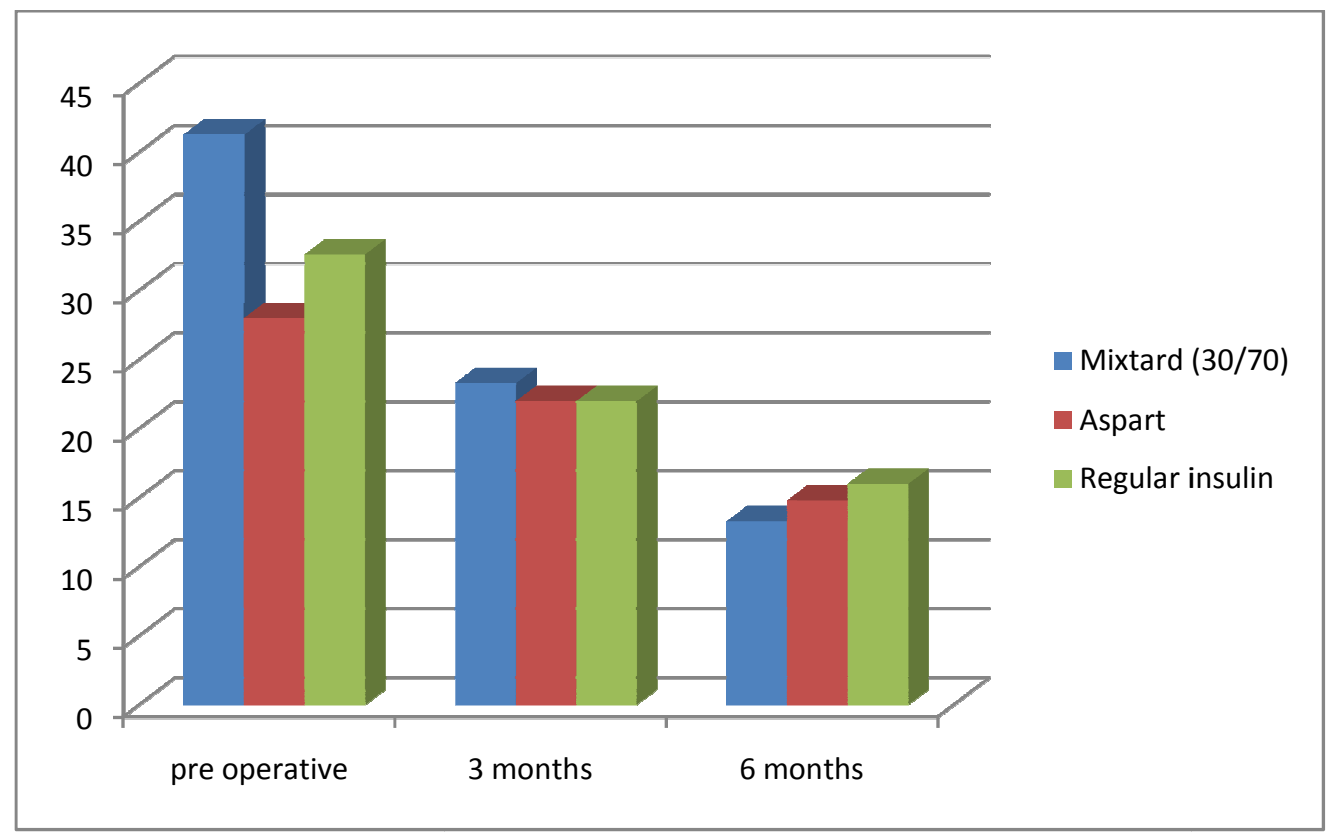

